\title{
Artefact
}

Techniques, histoire et sciences humaines

\section{Représenter, expérimenter, raconter : le Centre de réalité virtuelle à l'Université de Caen Normandie}

Representation, Experimentation, Narration : the Interdisciplinary Centre for Virtual Reality of Caen Normandy University

\section{Éric Leroy du Cardonnoy et Sophie Madeleine}

\section{(2) OpenEdition}

Édition électronique

URL : https://journals.openedition.org/artefact/10553

DOI : 10.4000/artefact.10553

ISSN : 2606-9245

Éditeur :

Association Artefact. Techniques histoire et sciences humaines, Presses universitaires du Midi

Édition imprimée

Date de publication : 7 octobre 2021

Pagination : 445-465

ISBN : 978-2-8107-0755-3

ISSN : 2273-0753

\section{Référence électronique}

Éric Leroy du Cardonnoy et Sophie Madeleine, « Représenter, expérimenter, raconter : le Centre de réalité virtuelle à l'Université de Caen Normandie », Artefact [En ligne], 14 | 2021, mis en ligne le 07 octobre 2021, consulté le 07 octobre 2021. URL : http://journals.openedition.org/artefact/10553 ; DOI : https://doi.org/10.4000/artefact.10553

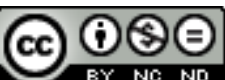

Artefact, Techniques, histoire et sciences humaines est mise à disposition selon les termes de la Licence Creative Commons Attribution - Pas d'Utilisation Commerciale - Pas de Modification 4.0 International. 


\title{
Représenter, expérimenter, raconter : le Centre de réalité virtuelle à l'Université de Caen Normandie
}

\author{
Éric Leroy du Cardonnoy et Sophie Madeleine
}

\section{Résumé}

Cet article présente le CIREVE (Centre interdisciplinaire de réalité virtuelle de l'Université Caen Normandie) son histoire et ses activités. Il retrace la construction du centre et de la salle immersive, des programmes développés à partir de la restitution du Plan de Rome (Ive siècle ap. J.-C.) ainsi que les nouvelles orientations prises autour des modélisations en géographie, médecine, neurosciences, littérature et histoire autour de trois notions clés : représentation, expérimentation, narration.

\section{Mots-clés}

immersion, interaction, Rome, réalité virtuelle, réalité augmentée

95 Éric Leroy du Cardonnoy et Sophie Madeleine, « Représenter, expérimenter, raconter : le Centre de réalité virtuelle à l'Université de Caen Normandie », Artefact, 14, 2021, p. 445-465. 


\section{Representation, Experimentation, Narration : the Interdisciplinary Centre for Virtual Reality of Caen Normandy University}

\section{Abstract}

This article deals with the history and activities of the CIREVE - Interdisciplinary centre for virtual reality - at Caen University. It shows the construction of the centre and the immersive room, the 3D-restitution of Rome ( $4^{\text {th }}$ century AD) as well as the new orientations around digital programs in geography, medicine, neurosciences, literature and history centered on three key-notions: representation, experimentation and narration.

\section{Keywords}

\section{Introduction}

En 1956, l'Université de Caen reçut comme cadeau pour célébrer sa reconstruction un objet à l'histoire et au trajet particuliers, qui est à l'origine de la naissance et de l'ancrage de la réalité virtuelle sur ce site normand : le «Plan de Rome » de Paul Bigot ${ }^{1}$. Paul Bigot (1870-1942), architecte d'origine normande, avait obtenu en 1900 le Prix de Rome en architecture et passé plusieurs années à la Villa Médicis à s'intéresser à la Rome antique, années pendant lesquelles il avait décidé de fournir comme "envoi de Rome " la réalisation d'un plan relief de la ville au Iv siècle après J.-C., en commençant par le Circus Maximus. Cette maquette de plâtre, qui dans sa version finale $-70 \mathrm{~m}^{2}$ - de 1942 représente à l'échelle $1 / 400$ les $4 / 5^{\mathrm{e}}$ de la Rome antique, dut en 1994 être restaurée et fut installée dans un nouveau bâtiment construit autour de cette maquette, la Maison de la Recherche 
en Sciences Humaines et Sociales ${ }^{2}$. C'est à ce moment que Philippe Fleury, Professeur d'études latines, décida d'utiliser les fonctionnalités de la réalité virtuelle (RV) pour pérenniser la maquette tout en prenant en compte les dernières données des recherches sur la Rome antique afin de " corriger » la restitution de Paul Bigot et d'en permettre une évolutivité au gré des nouvelles découvertes scientifiques. Ainsi il devenait possible de reconstituer, sans avoir à passer par des maquettes de plâtre (coûteuses en temps, argent, etc.), l'état des connaissances scientifiques à un moment donné, mais en même temps d'offrir par les potentialités de la réalité virtuelle une adaptation constante de données nouvelles à des réalisations déjà existantes, ce qui correspond tout à fait à l'esprit d'une science en mouvement, en évolution perpétuelle, et également ouverte, comme nous le verrons plus loin.

Le Centre interdisciplinaire de réalité virtuelle (CIREVE) de l'Université de Caen est né en 2006 sur la base du savoir-faire acquis depuis 1994 autour du projet Plan de Rome. Dans les années 90, le plan relief de Paul Bigot, communément appelé le "Plan de Rome $»^{3}$ fit l'objet d'une mise en valeur scénographique au sein de la Maison de la Recherche en Sciences Humaines de Caen. Une équipe de recherche ${ }^{4}$ s'attela dès lors à un projet d'envergure : restituer virtuellement la ville de Rome en 320 ap. J.-C., c'est-à-dire à la même époque que Paul Bigot, tout en reprenant les recherches sur l'architecture et l'urbanisme de la ville à nouveaux frais ${ }^{5}$. Il n'était en effet aucunement question de scanner la maquette de Paul Bigot pour la virtualiser, ce qui n'aurait eu que peu d'intérêt scientifique, mais bien de reprendre à zéro l'analyse des sources anciennes pour présenter un modèle scientifiquement à jour. L'expérience acquise au cours de ces années a permis de constituer un noyau d'enseignants-chercheurs, de chercheurs et d'ingénieurs qui ont progressivement mis en place une

\footnotetext{
2. Il ne reste aujourd'hui que deux maquettes en plâtre : l'exemplaire de Caen légué par P. Bigot à Henri Bernard, l'un de ses élèves qui en fit don à l'Université de Caen, et l'exemplaire de Bruxelles. En outre il existe également un plan partiel en bronze à l'Institut d'Art et d'Archéologie de Paris, Idem, p. 114-116.

3. Royo, 2006.

4. L'équipe "Plan de Rome " tire son nom de celui de la maquette de Paul Bigot, elle-même nommée ainsi en référence à la tradition des " plans reliefs " des villes fortifiées initiée en France au XVIII ${ }^{e}$ siècle par le ministre de Louis XIV, Louvois. Institutionnellement, l'équipe "Plan de Rome " fait partie de l'Équipe de Recherche sur les Littératures, les Imaginaires et les Sociétés " (ERLIS EA 4254) et elle s'appuie sur les moyens informatiques du Centre interdisciplinaire de réalité virtuelle (CIREVE) de l'Université de Caen Normandie.
}

5. Fleury, 2014, p. 109-124. 
méthodologie pour créer des modèles virtuels scientifiques avec accès aux métadonnées. Il semblait en effet indispensable de justifier les choix de restitution et de faire état des sources utilisées, qu'elles soient primaires ou secondaires ${ }^{6}$. Dès 2003, les premiers modèles interactifs ont vu le jour, ce qui a donné un nouveau degré de liberté à l'utilisateur : choix du parcours, possibilité de visiter Rome à la $3^{\mathrm{e}}$ personne pour mieux percevoir les échelles, etc. (https://rome.unicaen.fr/). Aujourd'hui, le modèle virtuel caennais est, avec celui de l'équipe américaine "Rome Reborn ", un des deux seuls modèles virtuels au monde à proposer une immersion dans la totalité de la ville de Rome ${ }^{7}$. Un contrat de partenariat a d'ailleurs été signé a d'ailleurs été signé avec "Rome Reborn ». Au fil du temps, la renommée de l'équipe caennaise a engendré des demandes émanant de tiers, collectivités locales ou laboratoires de recherche, pour restituer d'autres éléments patrimoniaux (ville de Caen avant les bombardements, domus de Vieux la Romaine, église de Thaon à différentes époques etc., et dernièrement la restitution des fortifications de Caen à différentes époques dont l'achèvement est prévu pour célébrer le millénaire de la ville en 2025). La chaîne de travail fonctionnait parfaitement, le personnel était formé, le matériel était opérationnel et l'idée a alors germé de partager ce savoir-faire avec les autres équipes de l'université de Caen Normandie, dans un horizon bien plus large que celui des seules sciences humaines et sociales. La pluridisciplinarité de cette université a permis de fédérer une dizaine d'équipes dans des domaines aussi variés que la neuropsychologie, les sciences du mouvement, l'informatique, la géographie et bien d'autres encore (https:// cireve.unicaen.fr/). En 2006, le CIREVE était né, augurant une montée en puissance de la réalité virtuelle mise au service de la recherche ${ }^{8}$.

C'est pourquoi les activités du CIREVE et ses réalisations seront présentées selon trois axes majeurs de la réalité virtuelle : dans un premier temps,

\footnotetext{
6. Fleury et Madeleine, 2009b.

7. Fleury et Madeleine, 2009a.

8. L'équipe actuelle du CIREVE est composée de cinq personnels titulaires : Sophie Madeleine, ingénieur de recherche habilitée à diriger des recherches, Leslie Decker, Maître de conférences habilitée à diriger des recherches en neuropsychologie, Nicolas Lefêvre, ingénieur d'études, François Legay, ingénieur de recherche, et Charlie Morineau, assistant ingénieur, auxquels s'ajoutent au gré des années des personnels en CDI, comme par exemple Axel Tiller, ingénieur d'études, des doctorants sur contrats ou des post-doctorants comme c'est le cas cette année avec Jérôme Nicolle, qui a soutenu sa thèse sur "Les automates dans l'Antiquité : sources, fonctions et restitution virtuelle " sous la direction de Philippe Fleury et Olivier Lezoray le 15 septembre 2020.
} 
nous nous intéresserons à la question de la représentation de réalités soit disparues, soit fortement endommagées, pour continuer par les potentialités offertes par la réalité virtuelle dans l'expérimentation - technique, combinatoire, conceptuelle - et terminer par la narration renouvelée grâce à la technique.

\section{La restitution virtuelle pour représenter}

Si l'usage le plus courant de la réalité virtuelle est celui du jeu vidéo, cette technologie a aussi été utilisée très rapidement pour représenter des mondes disparus ou fortement dégradés. Les détracteurs n’ont pas manqué pour souligner le danger de telles pratiques, qui figeaient une représentation hypothétique dans l'imaginaire collectif au risque de la voir se transformer en vérité historique. Le procès n'était d'ailleurs pas tant celui de la restitution virtuelle que celui de l'image ou peut-être même celui de la complétion de l'image par des données hypothétiques, souvent obtenues par analogie ${ }^{9}$. A cet égard, notre travail s'inscrit dans la lignée des célèbres envois de Rome, mais la réalité virtuelle ouvre de nouvelles perspectives, qui permettent de dépasser le stade de l'illustration ${ }^{10}$.

Une des utilisations les plus courantes de la restitution virtuelle se juxtapose partiellement à celui du dessin de restitution tel que le pratique par exemple J.-C. Golvin ${ }^{11}$. Il est possible de proposer une visualisation d'édifices disparus avec toutes les précautions oratoires à prendre pour expliquer qu'il s'agit d'une évocation, d'une hypothèse de restitution, quelle que soit

\footnotetext{
9. Golvin, 2005.

10. Le labex "Les passés dans le présent » est un projet de recherche collectif (2012-2019) coordonné par l'université Paris Nanterre. Il porte sur la présence du passé à l'ère du numérique et s'intéresse notamment aux dispositifs numériques de médiation du passé. Le projet « NimRoD : de Nimrud à Rome, découverte des bibliothèques antiques " est inscrit dans le socle scientifique du labex. NimRoD est au cour des problématiques de recherche et de valorisation du labex et implique un travail de modélisation s'appuyant sur les standards internationaux de représentation des données. Sur le plan scientifique, la modélisation en trois dimensions de la bibliothèque de l'Ezida de Nimrud constitue un outil essentiel à l'approfondissement de l'analyse architecturale, dans une perspective de publication. Sur le plan patrimonial, il s'agit d'un monument emblématique dont la restitution constitue un enjeu important. En termes de valorisation, le projet a un rôle à jouer auprès du grand public. La modélisation 3D de la bibliothèque de Nimrud s'inscrit dans le cadre de la collection Grands Sites archéologiques du ministère de la culture et de la communication, dont un des axes concerne le patrimoine du Proche-Orient en danger.
}

11. Golvin, 2008. 
la complexité des études réalisées en amont de la création de l'image. Nous ne prendrons ici qu'un seul exemple : celui de l'environnement immédiat du théâtre de Marcellus à Rome. Les figures 1 et 2 montrent l'état actuel et celui du IV siècle ap. J.-C. selon un point de vue comparable (Fig. XV et XVI, cahier couleur).

Ce théâtre, qui est aujourd'hui le mieux préservé de la Rome antique, se distingue aisément dans le tissu urbain de la capitale italienne, mais la disparition d'une partie de son élévation, de la totalité de ses gradins et de son mur de scène rend sa lecture difficile. Dans les années 1940, trois colonnes du temple d'Apollon ont été remontées devant le théâtre, mais là-aussi, la perception de l'état original est complexe. De surcroît, il serait erroné d'imaginer cet espace uniquement pourvu d'un temple et d'un théâtre. Les plus attentifs repéreront sur place les traces du temple de Bellone et imagineront qu'une structure imposante devait s'élever sur ce secteur, mais ce n'est pas tout : dans l'Antiquité, deux autres monuments très anciens qui n'ont laissé aucune empreinte visuelle étaient présents : la columna bellica et le perrirhanterion ${ }^{12}$. La perspective côté Champ de Mars septentrional, à l'arrière-plan du point de vue choisi, était enfin complètement bouchée par le portique d'Octavie, qui n'est plus guère visible aujourd'hui. Pour synthétiser, seule une poignée de spécialistes a probablement les clefs de compréhension nécessaires pour appréhender dans toute sa complexité l'agencement de ce secteur de Rome au IV siècle ap. J.-C. La restitution virtuelle permet non seulement de restituer scientifiquement ces différents monuments, mais aussi de les placer les uns par rapports aux autres, en prenant en compte le réseau viaire et les questions de relief $^{13}$. La restitution virtuelle est un excellent média pour partager l'image de ce que devait être ce secteur dans l'Antiquité, tout en permettant la multiplication des points de vue. Quelques pixels synthétisent ici la lecture et la synthèse de dizaines d'articles de recherche représentant des centaines de pages certes passionnantes, mais réservées à un panel de lecteurs réduits.

12. Voir la nocturne du Plan de Rome sur le théâtre de Marcellus pour plus de détails sur la fonction de ces édifices (https://www.youtube.com/watch?v=djWmCQqHKQg).

13. Le placement respectif des différents monuments de la Rome antique est réalisé grâce au Sistema Informativo Archeologico all'Atlante di Roma. Voir la communication de P. Carafa au colloque "Topographie et urbanisme de la Roudme antique » organisé à Caen en 2019 (conférence en ligne : https://rome.unicaen.fr onglet " Le projet de recherche "; publication en cours). 
Si nous regardons l'image de restitution dans le détail, nous voyons apparaître certains éléments en couleurs. L'intégration de la polychromie nous rapproche encore un peu plus de la réalité historique, comme nous allons le voir à travers deux exemples. Le théâtre de Marcellus est couvert d'un vélum, un système mécanique essentiellement constitué de toiles de lin et de pièces de bois, qui permettaient aux spectateurs de suivre les jeux sans être importunés par le soleil. Aujourd'hui, il ne reste rien de ce vélum, mais pourtant, pour le théâtre de Marcellus comme pour tous les édifices de spectacle romains d'une certaine importance, les voiles de protection contre le soleil étaient présents. Or, les textes anciens nous apprennent qu'ils étaient polychromes : Lucrèce parle des toiles jaunes, rouges et bleues qui colorent les vêtements des spectateurs installés dans les gradins ${ }^{14}$. Les toiles colorées figurées sur l'image de restitution permettent donc de sensibiliser un public, spécialiste ou non, sur la présence d'un système technique auxiliaire du spectacle dans l'antiquité romaine. Pour prendre un deuxième exemple, des acrotères en bronze doré sont restitués sur les frontons des temples de Bellone et d'Apollon. Là aussi, il s'agit d'un usage courant dans l'architecture religieuse ancienne et le détail est poussé jusque dans le calcul de leur hauteur: les infographistes suivent les préconisations de l'architecte Vitruve pour les restituer ${ }^{15}$.

Quand la restitution virtuelle est enfin accompagnée de personnages, comme celui qui se trouve sur le pronaos du temple de Bellone, la perception de l'échelle des édifices n'en est que facilitée : la monumentalité du temple est immédiatement lisible et parle bien plus qu'une indication simplement chiffrée, qui apparaît de surcroît dans les métadonnées associées au modèle. Ces dernières années, le développement de jeux vidéo toujours plus réalistes a permis de franchir une nouvelle étape pour donner de la vie aux restitutions virtuelles. Les personnages statiques étaient certes déjà précieux pour donner l'échelle, nous venons de le voir, mais aujourd'hui, il est possible de les animer, de même qu'un certain nombre d'autres éléments, des animaux ou des végétaux notamment. Les restitutions développées au CIREVE sont le fruit de travaux universitaires, qui visent à alimenter des recherches et à les partager. Où se situe la frontière entre l'habillage

14. Lucr. 4, 75-83 ; Madeleine, 2015, p. 43-68 ; Madeleine, 2017, p. 65-82.

15. Vitr. 3, 5, $12:$ :Les acrotères d'angle auront la même dimension verticale que le tympan mesuré dans son axe médian, ceux du sommet seront d'un huitième plus élevés que ceux des angles » (Texte établi, traduit et commenté par P. Gros, Paris, Les belles lettres, 2003 (1990). 
" gadget " de la restitution et l'intérêt de ces nouveaux outils pour la recherche et la pédagogie ? L'équilibre est délicat et il est nécessaire que les chercheurs ou enseignants-chercheurs qui produisent ces restitutions s'interrogent perpétuellement sur leur nécessité pour éviter tout écueil. Pour notre part, ces ajouts nous semblaient artificiels au départ, et nous défendions l'idée de terminer l'architecture de la ville avant de nous occuper de ces questions d'animation. Les ingénieurs de l'équipe ont souhaité réaliser des tests, pour nous montrer le potentiel de tels ajouts, et aujourd'hui, moyennant certaines précautions dans leur usage, nous sommes convaincus de la plus-value apportée. Prenons l'exemple de la restitution des soussols des thermes de Caracalla. Ils étaient principalement utilisés pour des considérations techniques : apporter le bois jusqu'aux fours, chauffer l'eau, etc. L'intérêt de parcourir des kilomètres de couloirs voûtés simplement revêtus d'enduit aurait été bien maigre, réduisant de ce fait la pertinence même de la restitution. Ce secteur de Rome est pourtant très riche : on sait que la largeur des couloirs permettait à des ânes tirant des charrettes de bois de se croiser, les archéologues ont retrouvé des bassins pour que les équidés puissent s'abreuver, etc. Les infographistes du CIREVE ont non seulement restitué ces sous-sols, mais ils ont aussi rendu leur fonction directement perceptible ${ }^{16}$ : les charrettes de bois se croisent, les ânes sont animés et ils s'abreuvent dans les bassins prévus à cet effet. Le bois destiné à être brûlé dans les thermes est représenté dans les espaces de stockage, de l'éclairage artificiel avec des lampes suspendues au mur a été ajouté, quand il est apparu que les seuls soupiraux attestés par l'archéologie ne donnaient pas suffisamment de lumière pour que des hommes travaillent dans ces sous-sols. Il est clair que la recherche n'a pas particulièrement progressé grâce à ce travail, mais d'un point de vue pédagogique, c'est un aspect souvent négligé de la pratique thermale qui a été mis en valeur, et qui est désormais compréhensible par le plus grand nombre ${ }^{17}$. Si d'autres équipes avaient déjà travaillé sur la restitution des pièces les plus connues des thermes de Caracalla (piscine à ciel ouvert, palestre, etc.), nous sommes à notre connaissance les seuls à nous être penchés sur la restitution virtuelle des sous-sols, avec notamment la présence d'un mithraeum qui permet

16. Voir la Nocturne du Plan de Rome dédiée au culte de Mithra : https://www.youtube.com/ watch?v=hSiXgvOjq7o\&list=PL8Pdu-rCrd3pECty8F0w4Ohd219bFINgG\&index=5

17. Un partenariat avec les éditions Hachette permet au CIREVE de diffuser ses restitutions virtuelles dans les manuels scolaires du secondaire, sous forme d'images dans les éditions papiers et de vidéos sur des plates-formes en ligne dédiées à l'enseignement. 
d'introduire la question des cultes orientaux à Rome (Fig. XVII, cahier couleur). Les thermes sont ainsi restitués dans toute leur complexité, dans une appréhension globale qui, seule, permet de toucher du doigt toutes les réflexions menées par les architectes au moment de leur construction.

Si la réalité virtuelle permet de représenter des édifices, des espaces de l'Antiquité comme les exemples précédents l'ont montré, elle permet également d'envisager des constructions ou des immeubles à venir ou en réaménagement : ainsi l'Université de Caen a mandaté le CIREVE pour réaliser la rénovation de son parc immobilier avec l'utilisation du logiciel BIM (Building Information Modeling). Un BIM a pour fonction de modéliser des données d'un bâtiment, d'une infrastructure afin d'en améliorer les performances énergétiques dans une réflexion de développement durable et d'économie raisonnée, de transformer la conception et l'exploitation des bâtiments en question. Il favorise ainsi les prises de décisions et améliore les performances du projet, mais modifie également les rôles des acteurs de la maîtrise d'œuvre. Ainsi l'un des bâtiments historiques de l'Université de Caen lors de sa reconstruction en 1957 est depuis 2018 objet d'une réhabilitation de grande ampleur - refonte de son utilisation, des aménagements intérieurs, de son infrastructure globale. Dans la même perspective, le CIREVE a intégré les données mises à disposition par la Direction de l'immobilier de l'Université de Caen afin de présenter et de communiquer sur le bâtiment du CREC (Centre de Recherches en environnement côtier) à Luc-sur-Mer, lui aussi en pleine rénovation.

\section{La réalité virtuelle pour expérimenter}

La restitution virtuelle peut devenir un outil d'expérimentation particulièrement performant quand elle évolue en réalité virtuelle. Ce concept de réalité virtuelle induit deux notions fondamentales : l'interaction avec le modèle et l'immersion dans le monde restitué. L'interaction peut prendre différentes formes, la plus répandue étant la liberté de circulation : l'utilisateur est actif dans la mesure où il choisit son parcours dans le modèle virtuel. Cette fonction permet d'estimer les temps de circulation, comme par exemple le temps nécessaire pour aller s'asseoir sur les gradins les plus hauts du Colisée, ce qui est difficile à calculer sans modèle virtuel puisque les gradins ont aujourd'hui disparu. Il peut également choisir une heure de la journée pour voir évoluer l'ambiance d'un édifice en fonction de 
l'intensité lumineuse qu'il reçoit. S’il le souhaite, l'utilisateur peut accéder aux métadonnées qui l'informeront sur le degré de certitude de la restitution, qui peut aller du très hypothétique à la quasi-certitude dans certains cas. Il est aussi possible de voir comment un édifice a évolué au cours de l'histoire en proposant différentes dates pour l'immersion. Dans le modèle virtuel de la Rome antique développé à Caen, il est possible de visiter les enclos de vote de l'époque républicaine, les Saepta Iulia, puis de déambuler en leur sein à l'époque impériale, date à laquelle ils ont été transformés en marchés de luxe. L'évolution de l'architecture en fonction des différents régimes politiques est clairement visible. Le modèle virtuel devient dès lors un support pour expliquer des notions qui dépassent le strict cadre architectural, comme par exemple celui la propagande impériale ou de l'architecture au service du pouvoir.

Pour que l'on puisse parler d'immersion, il faut que l'utilisateur ait la sensation d'être dans le monde virtuel, qu'il passe de l'autre côté de l'écran pour reprendre une expression canonique. Cette opération est possible avec des moyens complexes et onéreux, comme une salle de réalité virtuelle, mais aussi avec des outils plus simples comme un casque de réalité virtuelle, une tablette ou un smartphone. L'application Roma in Tabula, développée au CIREVE, offre toutes ces possibilités. Elle est gratuite et téléchargeable sur PC, smartphones ou tablettes Android ${ }^{18}$. Sur les supports Android, cette application offre deux types de visites pour un certain nombre de monuments de la Rome ancienne. La première option consiste à choisir une visite en réalité augmentée : elle permet d'avoir une vision globale du bâtiment, vu de l'extérieur. Ce dernier apparaît avec son élévation complète et ses couleurs au-dessus du plan au sol réalisé par les archéologues. Pour entrer dans le monument, il faut passer en mode réalité virtuelle. L'utilisateur de trouve alors dans l'édifice, avec une liberté totale de circulation puisqu'il peut se déplacer à l'aide de joysticks virtuels. Les deux modes permettent à chaque instant d'accéder aux métadonnées justifiant les choix de restitution. Si cette immersion offre déjà tout un panel de possibilités, la meilleure solution pour visiter un monde restitué à l'échelle 1, en ayant un fort sentiment de présence dans cet environnement de synthèse, reste la salle de réalité virtuelle communément appelée le CAVE. 
Le CAVE du CIREVE est une partie de l'extension de la MRSH de l'Université de Caen et un outil particulièrement utile pour créer ce sentiment de présence, que ce soit dans des lieux réels - disparus ou menacés - ou imaginaires. Il dispose en effet d'une configuration unique en France puisqu'il conjugue le CAVE à d'autres instruments indispensables pour visiter un monde à l'échelle 1. Achevé fin 2015, il a été inauguré officiellement en 2017. L'intérêt de cette extension réside dans deux points qui en font son unicité : tout d'abord dans le fait que la définition de l'équipement a été effectuée avant celle de l'immobilier, autrement dit le projet architectural a été construit autour de l'équipement, permettant ainsi de faire passer les besoins de recherche avant la conception immobilière. Les besoins des équipes ayant été pris en compte très en amont (dès 2003), ils ont pu être revus jusqu'au dernier moment. Ainsi une équipe avait besoin d'une grande longueur d'écran pour étudier le problème des traversées de route, il a été construit une salle correspondant à cette demande et ainsi a vu le jour la plus grande salle immersive de France en milieu universitaire $(10 \mathrm{~m}$ de long) (Fig. 1). Une autre équipe avait besoin d'un tapis de marche de type GRAIL ${ }^{19}$ : Caen est ainsi le seul lieu de France où un tapis de ce type est installé dans un si grand $\mathrm{CAVE}^{20}$. D'autre part cette extension réunit autour d'un même ensemble de calculateurs deux outils nécessaires à la réalité virtuelle : un amphithéâtre pour des présentations collectives et un CAVE pour des expérimentations individuelles. Cette configuration a un double intérêt : elle n'exige qu'une seule maintenance de logiciels et surtout elle offre la possibilité de retransmettre en direct une expérimentation faite avec un seul sujet à 150 personnes en même temps.

19. Il s'agit d'un tapis de marche à double plateforme qui permet une analyse du mouvement dans des conditions de temps réel ; il permet également d'analyser tout mouvement, déplacement de chaque pied séparément ; enfin la vitesse du tapis de marche est donnée par le sujet de l'expérience lui-même - contrairement aux tapis de course/marche traditionnels.

20. Des travaux, terminés en septembre 2020 et entrepris grâce aux financements de l'Union Européenne, de la Région et de l'Université de Caen, ont optimisé le tapis Grail et l'utilisation de la salle immersive en permettant d'escamoter le tapis dans le sol, réduisant d'une part, les manipulations de l'appareil - qui pèse $1 \mathrm{~T}$ - et d'autre part, les interférences avec le monde non virtuel - en effet jusqu'ici les sujets qui se trouvaient sur le tapis Grail à $50 \mathrm{~cm}$ au-dessus du niveau du sol ne pouvaient pas être "totalement » immergés, car le haut du champ de vision était impacté (le regard passait au-dessus du haut de l'écran). La mise en place d'une plate-forme élévatrice pour mettre les bandes de roulement du tapis GRAIL au niveau du sol de la salle immersive quand son utilisation est requise a l'avantage de libérer ainsi l'espace qu'il occupait devant la salle de contrôle. La fosse est alors recouverte d'un écran escamotable et la totalité du sol de la salle de réalité virtuelle est disponible pour recevoir une projection. 


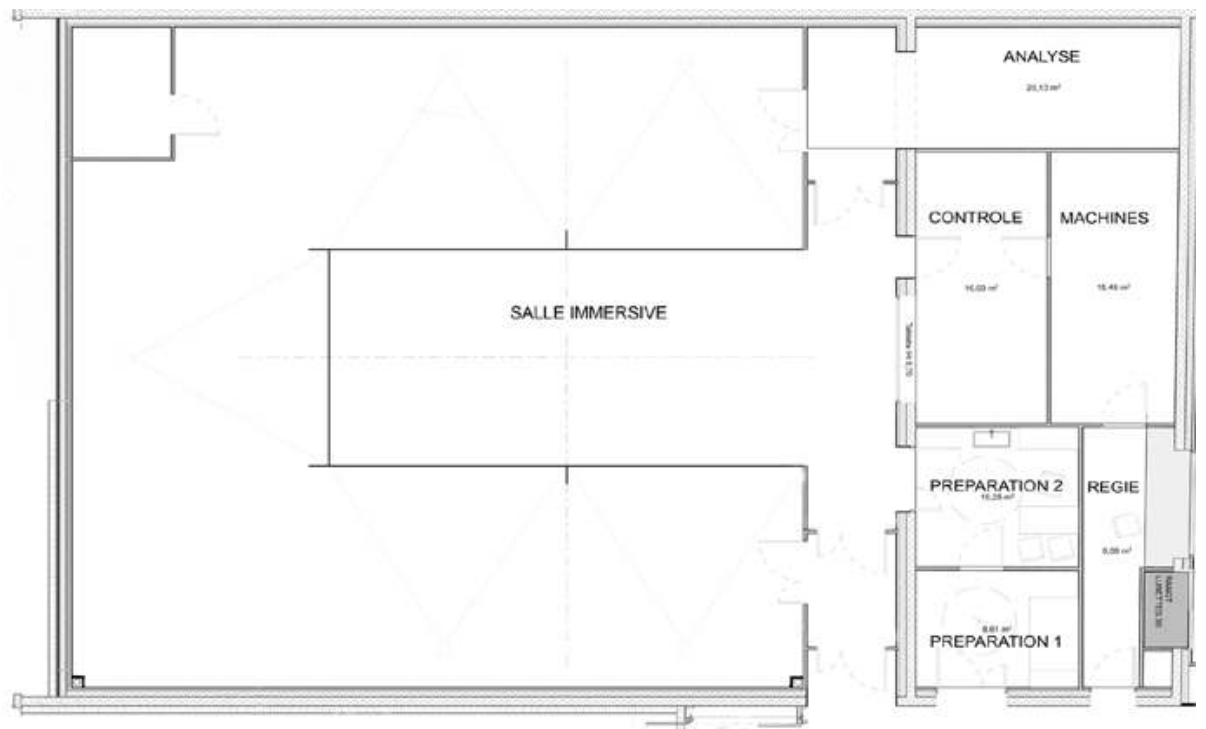

Fig. 1. - Plan de la salle de réalité virtuelle de l'Université de Caen

La configuration de la salle immersive est la suivante :

- Écrans :

- 2 écrans latéraux $(9,60 \mathrm{~m} \times 3 \mathrm{~m})$

- 1 écran de face $(4,80 \mathrm{~m} \times 3 \mathrm{~m})$

- 1 écran de sol $(9,60 \mathrm{~m}$ x 4,80 m)

- Projection :

- 8 projecteurs 12000 lumens, 4K UHD @ 60Hz / 2,560 x 1,600 @ $120 \mathrm{~Hz}$

- Tracking :

- 13 caméras Caméras VERO2.2 (2,2 millions de pixels - $330 \mathrm{~Hz})$

- Oculomètre Dikablis Ergonners

Elle dispose également de deux salles de préparation pour les sujets d'expérimentation.

À cela s'ajoutent

- une salle d'analyse

- une salle de contrôle

- une salle des machines

- un tapis roulant instrumenté M-GAIT (Motekforce Link). Ce tapis GRAIL peut être escamoté dans une fosse afin de libérer l'ensemble de l'espace de la salle. 
Enfin une régie - à droite de l'illustration - permet de retransmettre les expérimentations dans l'amphithéâtre accolé à la régie. Cet amphithéâtre est doté d'un écran permettant la restitution 3D simultanée des expériences en cours, soit du point de vue du sujet, soit du point de vue du chercheur contrôlant le processus. Ainsi la capacité de la salle immersive - limitée à 5 personnes maximum - peut de la sorte être dépassée (Fig. XVIII, cahier couleur).

Cet équipement a permis d'élaborer, voire de réaliser différents projets qui peuvent être regroupés sous trois catégories représentant diverses approches inter- et transdisciplinaires dont seuls quelques exemples seront donnés ci-après ${ }^{21}$ :

1. la prospective en géographie physique et humaine

2. la locomotion humaine, la motricité et la cognition

3. la présence, la réalité et le réalisme

1. Le premier projet, de géographie physique et humaine, porté par Stéphane Costa, Université de Caen Normandie, Laboratoire LETG Caen Géophen, se décline sous deux formes complémentaires, RAIV-Cot et REVE-Cot (2018-2021), qui portent sur la géographie du littoral et ont pour but de présenter les conséquences d'une tempête - du type de celle de 1990 - avec une élévation du niveau de la mer de $1 \mathrm{~m}^{22}$. Le but de ces deux projets, au-delà des restitutions virtuelles de villes côtières et de leur littoral, est de parvenir à une appropriation de l'aléa et à une exploration du littoral de demain grâce à la réalité virtuelle dans une démarche prédictive à l'attention des édiles des villes concernées par une simulation des fluides des sites choisis. Un élément non négligeable de cette immersion, le son, a été intégré dans ces projets particuliers, grâce à un ingénieur d'études du CIREVE, afin d'en tester sa validité et ses effets : ainsi la restitution virtuelle devient un outil de pronostic, de sensibilisation et de réflexion prospective sur les questions d'urbanisme, de politique publique de la part des collectivités locales ainsi que sur le sujet de l'aménagement du territoire dans une démarche qui rassemble non seulement des scientifiques - issus de diverses disciplines (sciences physiques, sciences naturelles, sciences humaines) - mais aussi des décideurs et les populations concernées. Ce

21. Il est nécessaire ici de mentionner le projet PROSOM-K (portant sur le stress et la mémoire), débuté fin septembre 2020 en collaboration avec la plateforme Cycéron, qui est désormais achevé. 22. Cf. https://www.youtube.com/watch?v=Gy6tmLZYa9w 
projet contribue de la sorte au mouvement de la science ouverte et participative par la mise à disposition d'un outil performant qui permet à tous les acteurs d'apporter leur contribution dans le débat qu'entraînent les conséquences de l'élévation régulière du niveau de la mer. Ces différents acteurs peuvent ainsi éprouver les conséquences du réchauffement climatique pour les villes concernées - à ce jour quatre sites correspondant à des aléas différents ont été modélisés : Quiberville-Sainte-Marguerite (SeineMaritime, Normandie), Étretat (Seine-Maritime, Normandie), Villerssur-Mer (Calvados, Normandie) et Lancieux-Ploubalay (Côtes d'Armor, Bretagne) - sans engendrer de "désastres matériels".

2. Dans la seconde catégorie se trouvent deux projets portés Leslie Decker, Université de Caen Normandie, laboratoire COMETE UMR-S 1075 INSERM. Le premier projet, Vitalised, court sur les années 2015-2022 et vise à mieux caractériser le Syndrome d'Ehlers-Danlos de type hypermobile (SEDh) et à évaluer l'efficacité d'une stratégie thérapeutique non médicamenteuse, associant la suppléance proprioceptive via le port de vêtements compressifs et la reprogrammation sensorimotrice sur plateforme motorisée, dans le traitement de la déficience sensori-motrice associée au syndrome en question. L'étude prévoit l'inclusion de quarante patients SEDh et quarante sujets contrôles qui seront suivis sur une année divisée en trois périodes de 4 mois : la première période sera consacrée à l'homogénéisation de la prise en charge médicale, la seconde portera sur la reprogrammation sensorimotrice (12 séances) couplée au port (6 heures par jour) de vêtements compressifs ou peu compressifs, enfin la dernière période sera dédiée à l'arrêt de la stratégie thérapeutique et à l'évaluation de la rémanence. Les patients seront évalués à chaque temps de l'étude à partir de tests, de mesures comportementales en réalité virtuelle, et de questionnaires à domicile portant sur les symptômes et la qualité de vie. Dans ce contexte, la salle immersive dont dispose le CIREVE permet d'effectuer les expérimentations présentées, sans aucun risque physique pour les patients et tout en respectant l'intégrité physique des sujets ; les salles de préparation ont été aménagées pour précisément pouvoir prendre en compte les besoins éventuels des patients (nécessité de pauses et de repos par exemple) (Fig. XIX, cahier couleur).

Le second, Présage, commencé en 2019 se terminera en 2023 : il s'agit d'étudier le syndrome pré-démentiel du risque cognitivo-moteur du sujet âgé, pour savoir s'il s'agit d'un syndrome de déconnexion cérébrale du 
réseau exécutif central. Cette étude s'intéresse à l'étiologie du syndrome mentionné, un syndrome pré-démentiel qui se caractérise par un déficit cognitif subjectif et une vitesse de marche ralentie, et qui multiplie par trois le risque de développer une démence - l'utilisation du tapis Grail est ici tout à fait approprié à ce type d'expérience par les garanties qu'il offre ${ }^{23}$. L'hypothèse de recherche est que ce syndrome pourrait trouver son origine dans un défaut du contrôle exécutif affectant les domaines cognitifs et sensori-moteurs. À cette fin, des mesures de connectivité cérébrale et comportementales, en réalité virtuelle et à domicile, sont collectées chez des sujets présentant le syndrome en question et des sujets contrôles. Les sujets ayant le syndrome seront revus à deux ans dans le but d'identifier une évolution vers un syndrome démentiel débutant.

Ces deux projets utilisent chacun des pans différents, mais complémentaires, des capacités de la salle immersive, à la fois le côté expérience de motricité dans un contexte spécifique sans risque pour les sujets-témoins, d'autre part les possibilités de collecte de données - ici dans le domaine cognitif et proprioceptif - afin de proposer des prises en charge ou des soins non médicamenteux sans risques pour les sujets. Ils permettent également d'engranger un nombre de données qui peuvent être éventuellement réutilisées, et ils participent à la formation des étudiants participant aux expérimentations par des enseignants-chercheurs réalisant ainsi le plus concrètement l'adossement de la formation à la recherche et le fait que la recherche alimente l'enseignement au plus près (Fig. $\mathbf{X X}$, cahier couleur).

3. Le troisième volet, que nous aimerions ici considérer, est une orientation relativement nouvelle au sein du CIREVE, qui ressortit à une interrogation méta-réflexive des méthodologies mises en œuvre et à l'un des points névralgiques des restitutions en réalité virtuelle, à savoir une interrogation sur la question de la présence en réalité virtuelle d'une part et de la notion de « réalisme » dans le champ de la réalité virtuelle d'autre part. Le premier point étant relativement récent, il est peut-être encore trop tôt pour en faire ici une plus ample présentation ; il a fait l'objet d'une thèse soutenue fin 2020 à l'Université de Caen et nécessite un approfondissement de cet axe de recherche ${ }^{24}$.

23. Le tapis escamotable peut être équipé de rampes sur lesquels les sujets peuvent prendre appui et un harnais accroché depuis les cintres de la salle évite tout risque de chute possible.

24. "Le sentiment de présence en réalité virtuelle : rôle modérateur des facteurs humains sur la performance " (Arthur Maneuvrier) thèse soutenue le 15 décembre 2020. http://www.unicaen.fr/ recherche/mrsh/erlis/6572 
Parallèlement à cette thèse qui tente d'appréhender le sentiment de présence, un programme s'est mis en place sur la représentation de la "réalité " en littérature avec utilisation de la réalité virtuelle, qui au-delà des simples questions matérielles, vise à s'interroger sur la notion de réalisme en littérature et en réalité virtuelle : y a-t-il une différence fondamentale entre les deux ? Et si oui, de quelle nature est-elle ? Porté par un Professeur de littérature et civilisation germaniques, il s'agit entre autres d'essayer de reconstituer dans un premier temps un "espace imaginaire ", la Maison des roses du roman L'Arrière-saison (1857) d'Adalbert Stifter, qui constitue le noyau de l'ouvrage puisque presqu'un quart du roman est consacré à de longues descriptions de ce bâtiment, tout en réfléchissant par ce biais à la notion de "réalisme " aujourd'hui au vu des avancées techniques de la réalité virtuelle. Les allers et retours entre l'univers de l'imaginaire et l'univers du réel sont un thème ancien de la fiction, que le chemin se fasse dans le sens du réel vers imaginaire ou de l'imaginaire vers le réel. Les lieux ont toujours joué un rôle important dans ce passage, car ils permettent d'ancrer les univers de fiction dans le réel. En retour, les lieux et les paysages créés par les artistes transforment la manière dont les espaces réels sont perçus en leur ajoutant de nouvelles dimensions. À une époque où les images des lieux deviennent un facteur majeur de leur développement, cette connexion entre espaces imaginaires et espaces réels a pris une dimension démesurée, nécessaire pour appréhender les pratiques spatiales des lieux. Selon Marc Augé, « dans l'espace urbain et l'espace social en général, la distinction entre réel et fiction devient floue ${ }^{25}$ ». Mais cette thèse de la fictionnalisation du réel n'épuise en rien l'ensemble des relations complexes qu'entretiennent lieux réels et lieux imaginaires. Dans la continuité de deux ouvrages déjà publiés ${ }^{26}$, ce programme se propose, grâce au savoirfaire des membres de l'unité de recherche ERLIS EA 4254, spécialisés dans le numérique et la restitution virtuelle ainsi que la réalité augmentée, de modéliser certains lieux de fiction afin de visualiser les questions d'occupation de l'espace et de métaphorisation de l'écriture. Il s'agit par conséquent d'un programme qui utilise les compétences, acquises par l'équipe ERLIS et le CIREVE à travers le Plan de Rome, pour s'interroger sur les implications d'une représentation de l'espace sur le lecteur, le spectateur, ou tout simplement le citoyen de notre monde du $\mathrm{xx}^{\mathrm{e}}$ siècle, et de revenir sur des

25. Augé, 1997, p. 169.

26. Leroy du Cardonnoy, 2013 ; Czerny et Leroy du Cardonnoy, 2015. 
catégories que la réalité virtuelle remet en cause, comme par exemple celle de "réalisme », et les revisiter sur le plan théorique également.

\section{La réalité virtuelle pour raconter ${ }^{27}$}

Comme annoncé plus haut, la réalité virtuelle permet également de moderniser les techniques et perspectives de narration, non seulement en recherche fondamentale mais également dans une perspective de diffusion de la connaissance et de vulgarisation. Elle implique notamment une réflexion sur le contexte de diffusion, mais également sur les techniques de scénarisation qui amènent à spatialiser l'écriture ${ }^{28}$. Il s'agit en effet d'un renouvellement du genre dont les interactions avec la narration constituent un déf tant pour les concepteurs que pour les utilisateurs. Trois projets en cours actuellement s'inscrivent dans cette perspective.

Tout d'abord, l'étude intitulée « les fortifications de la ville de Caen : l'enceinte urbaine, le château et les clos abbatiaux, $\mathrm{XI}^{\mathrm{e}}-\mathrm{XXI}^{\mathrm{e}}$ siècles. Topographie historique, recherche archéologique et modèles numériques " financée par la ville de Caen et le CIREVE s'inscrit dans le cadre des festivités prévues pour le millénaire de la ville en 2025 et a débuté en 2021. Les recherches archéologiques sur le château et son environnement amènent à s'interroger sur le lien entre la forteresse et le paysage urbain qu'elle domine. L'articulation du système de défense préservé (le château) avec la fortification urbaine disparue (les remparts et portes de la ville) est une des questions posées pour la compréhension de la topographie historique et d'un système de défense en profondeur dont le château est la place forte et le donjon l'ultime réduit. La chronologie relative des éléments du système défensif et son évolution ont fait l'objet d'études archivistiques, de plans et tracés schématiques, de relevés archéologiques et études du bâti partielles sur des sections du rempart. Ces données modélisées doivent déboucher sur une restitution au public dans le cadre d'une narration de l'histoire de la ville : la réalité virtuelle devient l'un des outils qui permet aux citoyens de s'approprier l'histoire de leur environnement, existant ou disparu, par la production d'un discours porté et présenté par les images de réalité virtuelle.

27. Ryan, (2001) 2015.

28. Morriet, 2019. 
La deuxième action, qui existe maintenant depuis 2006, s'intitule les Nocturnes du Plan de Rome et forme le volet narratif des travaux sur le Plan de Rome ; elle permet de réunir chaque premier mercredi du mois environ 250 personnes dans l'amphithéâtre Pierre Daure de l'Université de Caen pour présenter l'une des activités de restitution virtuelle et contribuer ainsi à la vulgarisation des recherches effectuées au sein du CIREVE dans le cadre de la stratégie de science ouverte. De plus, une fois par an, un chercheur reconnu est invité à venir présenter une partie de ses recherches en lien avec la restitution du Plan de Rome. Les Nocturnes se trouvent donc à l'interface de la recherche et de la diffusion de celle-ci sous la forme d'une présentation et d'un débat. Depuis 2014 les séances font l'objet d'une captation qui est retransmise en directe sur la chaîne youtube et d'un débat avec le public après la projection par des questions posées soit dans la salle soit en ligne - la modération étant assurée par un membre du CIREVE. Il s'agit ainsi d'une scénarisation de la recherche à visée pédagogique qui a conquis une audience de plus en plus nombreuse.

Le dernier projet dans cette orientation est la reconstitution de la ville de Vienne (Autriche) au moment du Congrès de Vienne (octobre 1814juin 1815), et ce afin de mieux comprendre la logistique mise en place à l'époque dans l'espace urbain, les aménagements effectués, etc. pendant les neuf mois des négociations, afin aussi entre autres de visualiser les déplacements dans l'espace et reconstituer éventuellement des lieux aujourd'hui disparus grâce à des plans, des reproductions de l'époque ${ }^{29}$. Ce projet en est encore à ses débuts, car il nécessite d'établir des contacts étroits avec des historiens, majoritairement étrangers, spécialistes de la question - Vienne dispose en effet d'archives précises sur la topographie, les bâtiments de la ville à l'époque du congrès après la seconde numérotation des bâtiments instaurée en 1795 - et de trouver des fonds nécessaires pour sa réalisation ; on pourrait dire qu'il s'agit d'une facette plus moderne de ce qui a été initié par les recherches sur le Plan de Rome. Depuis la tenue du Congrès, cette manifestation unique a fasciné les historiens, les diplomates, les économistes mais également les romanciers et auteurs de théâtre et a été l'objet de projections contradictoires, qu'une mise en récit à partir des données scientifiques par la réalité virtuelle peut permettre de relativiser, d'infirmer,

29. Des conventions seront établies avec différentes instances publiques - archives, musées, etc. afin de pouvoir utiliser les sources les plus fiables dans cette reconstitution. 
voire de confirmer, mais peut-être avant tout de mettre en avant les déterminations politiques, économiques et spatiales de cet événement.

\section{Conclusion}

Le CIREVE, nous l'avons vu, est un plateau technique de l'Université de Caen et de ce fait a pour vocation d'être au service des chercheurs quelle que soit leur discipline. Son développement autour du Plan de Rome explique l'accent longtemps porté sur l'antiquité romaine du $\mathrm{IV}^{\mathrm{e}}$ siècle ap. J.-C., mais depuis presque dix ans maintenant, il a su opérer un changement de paradigme épistémologique afin de mettre en place une interdisciplinarité fructueuse. Par différents programmes - géographie, neurologie, histoire, littérature par exemple - l'équipe du CIREVE a montré que son dynamisme allait bien au-delà de ce qui existait et a su accroître les compétences de son équipe en stabilisant ses effectifs sur des postes pérennes et en renforçant ses domaines de compétences. Représenter et expérimenter restent les grandes tâches auxquelles le CIREVE s'attèle, même si raconter reste l'un des points fondamentaux dans la transmission du savoir qu'il s'est toujours donné.

Depuis quelques années, comme l'ont montré les différents projets et études menés par le CIREVE, une nouvelle orientation de développement des recherches s'est fait jour : des interrogations plus générales, sur la position du "spectateur » qui devient, dans une mesure limitée, « acteur » dans ce monde nouveau, sont venues élargir le champ des recherches, signalant ainsi la vitalité et le dynamisme de l'équipe : le changement radical de paradigme quant au positionnement physique (spatio-temporel) du sujet des expérimentations mais aussi du spectateur engendre un positionnement émotionnel différent. Par ailleurs, la question fondamentale soulevée par la réalité virtuelle du sentiment de présence et de réalité par l'adaptation du sujet et de ses perceptions et représentations ouvre de nouvelles perspectives que certains programmes auront à cœur de mettre en avant.

L'ouverture à d'autres domaines scientifiques montrent dans quelle mesure la réalité virtuelle est devenue une partie intégrante du monde de la recherche, qui converge notamment vers la question de la modélisation, ce qui est peut-être en dernier ressort le point de rencontre de toutes les disciplines qui participent aux actions présentées dans cette contribution 
et mériterait certainement une réflexion plus poussée sur sa nature, ses fonctions épistémologiques et ses limitations.

\section{Bibliographie}

Augé Marc, L'Impossible voyage. Le tourisme et ses images, Éditions Payot \& Rivages, Paris, 1997 (Coll. Petite bibliothèque n 214).

Czerny Boris et Leroy du Cardonnoy Éric (dir.), Lieu(x) d'écriture et écriture de lieu(x), Topographie du réel et de l'imaginaire, PUC, Caen, 2015 (Coll. Miscellanea).

Fleury Philippe, "Le plan de Rome de Paul Bigot. : De la maquette en plâtre de Paul Bigot à la maquette virtuelle de l'Université de Caen ", Civiltà Romana, $\mathrm{n}^{\circ}$ 1, 2014, p. 109-124.

Fleury Philippe et Madeleine Sophie, "Interactive Visit of the city of Rome in the fourth century A.D. ", dans Making History Interactive. Computer Applications and Quantitative Methods in Archaeology (CAA: proceedings of the 37th international conference, Williamsburg, Virginia, United States of America, March 22-26, 2009), B. Frischer, J. Webb Crawford, D. Koller (dir.), Archaeopress, 466 Oxford, 2009a, p. 67-75.

Fleury Philippe et Madeleine Sophie, "L'accès aux sources anciennes sur le modèle virtuel de la Rome antique ", Schedae, 22, 2009b, p. 5-18.

Royo Manuel, Rome et l'architecte : conception et esthétique du plan-relief de Paul Bigot, Presses Universitaires de Caen, Caen, 2006.

Golvin Jean-Claude, "L'image de restitution et la restitution de l'image ", cours en ligne sur la restitution à Tunis, 2005, https://jeanclaudegolvin.com/ cours-ligne-de-jean-claude-golvin-tunis-2005 I

Golvin Jean-Claude, Rome antique retrouvée : l'Urbs, les sanctuaires, les spectacles, les palais, Ostie, Villa Hadriana, Palestrina, Villa de Tibère, Errance, Paris, 2008.

Leroy du Cardonnoy Éric (dir.), Cités imaginaires, Ed. Petra, Paris, 2013

Madeleine Sophie, "La restitution d'un vélum sur le théâtre de Pompée ", actes du colloque international La technologie gréco-romaine entre restitution et reconstitution. Lire entre les lignes, mettre en les mains, Université de Caen BasseNormandie, 10-12 mars 2010, Presses Universitaires de Caen, Caen, 2015.

Madeleine Sophie, "Essai de typologie du vélum sur les théâtres romains » dans Autour des machines de Vitruve. L'ingénierie romaine : textes, archéologie et restitution, Presses universitaires de Caen, Caen, 2017. 
Morriet Oriane, "Concevoir et écrire pour la réalité virtuelle : nouvelles compétences, approches et techniques de scénarisation " in Mise au point (en ligne) L'évolution numérique des métiers du cinéma et de l'audiovisuel. Transformations, renouvellements et nouvelles qualifications, 12/2019, URL : http://journals.openedition.org/map/3825

Nicolle Jérôme et Madeleine Sophie «Roma in Tabula. La Rome virtuelle à disposition de publics pluriels ", In Situ. Revue des patrimoines, 42, 2020, mis en ligne le 13 juillet 2020, URL : https://journals.openedition.org/insitu/27236 ; DOI : $10.4000 /$ insitu. 27236

Ryan Marie-Laure, Narrative as Virtual Reality 2. Revisiting Immersion and Interactivity in Literature and Electronic Media, J. Hopkins University Press, Baltimore, (2001) 2015.

\section{L'auteur et l'autrice}

Éric Leroy du Cardonnoy est professeur de littérature et civilisation de langue allemande et vice-président recherche à l'Université de Caen Normandie. Il étudie principalement la littérature et culture de l'Autriche du xIx ${ }^{\mathrm{e}}$ siècle, en particulier F. Grillparzer et A. Stifter. Au sein du CIREVE, il a mis en place un programme de modélisation de lieux littéraires. Il a publié « Der arme Spielmann : ein politischer Text ? », Jahrbuch der Grillparzer-Gesellschaft 3. Folge, Band 28 (2019-2020), Vienne, Verlag Lehner, 2020, p. 7-27. Il est co-directeur de la collection Studia Habsburgica à Épure, maison d'édition de l'Université de Reims. Il est rédacteur en chef et éditeur (avec Alexandra Merle) de la revue électronique Histoire culturelle de l'Europe, http://www.unicaen.fr/mrsh/hce/. Contact : eric.leroyducardonnoy@ unicaen.fr

Sophie Madeleine est ingénieure de recherche en analyse de sources anciennes HDR, directrice du Centre interdisciplinaire de réalité virtuelle de l'Université de Caen Normandie (CIREVE). Elle est spécialiste de la topographie et de l'architecture de la Rome antique, de même que des techniques de l'Antiquité. Ses recherches portent principalement sur les théâtres et sur l'utilisation des vélums dans l'Antiquité. Au sein du CIREVE, elle est co-responsable du projet de restitution virtuelle de la Rome du ive siècle ap J.-C. (https://rome.unicaen.fr/). Contact : sophie.madeleine@unicaen.fr 